\title{
Imaging the joint and enthesis: insights into pathogenesis of psoriatic arthritis
}

\section{McGonagle}

The distinct radiographic features of psoriatic arthritis ( $\mathrm{PsA}$ ) help confirm it as a distinct entity from rheumatoid arthritis and highlight some unique non-synovial based disease imaging features. The advent of magnetic resonance imaging and a better understanding of joint microanatomy including the complexity of joint entheses provide a unifying anatomical and biomechanical concept that links disease at the apparently disparate sites of involvement in $\mathrm{Ps} A$, including the synovium, the enthesis, the bone and the periosteum. These findings suggest a reason for the localisation of disease to skeletal sites that are subject to repeated mechanical stressing.

$\mathrm{P}$ soriatic arthritis (PsA) encompasses several subgroups wherein the common denominator is an association with skin psoriasis. ${ }^{1}$ The spectrum of joint inflammation in PsA is great, ranging from axial to peripheral disease, synovial and adjacent soft tissue inflammation, enthesitis, osteitis, new bone formation and severe osteolysis, and overlaps of all of these. ${ }^{2}$ The distinguishing radiographic features of PsA are enthesis related changes including entheseal new bone formation, periostitis, entheseal erosions, and diffuse bone based pathology. ${ }^{3}$ Other imaging modalities such as scintigraphy, arthroscopy, ultrasonography, and magnetic resonance imaging (MRI) yield further information on these unique features of PsA and indicate that they are much more common than evident on clinical grounds.

\section{THE ENTHESIS ORGAN AND FUNCTIONAL ENTHESIS IN PsA \\ Soft tissue changes}

A prerequisite for understanding of the imaging findings in PsA hinges on an understanding of the relevant joint anatomy. This has been reviewed in detail elsewhere. ${ }^{4}$ The enthesis is the insertion of ligament, tendon, or joint capsule to bone, and enthesitis is a cardinal feature of the spondyloarthropathies (SpA). ${ }^{5}$ Enthesitis has been viewed as focal insertional inflammation. When the enthesis centred model for PsA was proposed, the nature of the pathology associated with enthesitis was only just being elucidated. ${ }^{26}$

On MRI, the SpAs are associated with extensive inflammatory changes at a considerable distance from the enthesis, illustrated at the plantar fascia and Achilles' tendon where enthesitis is associated with marked osteitis or synovitis in the immediately adjacent tissues. ${ }^{7}$ Achilles' tendon is comprised of not just the insertions but also prominent adjacent fibrocartilages that function to dissipate stress and protect the joint. It is now recognised that the enthesis represents a group of functionally linked tissues that should be viewed as unique interacting anatomical structures, properties that led anatomists to view the entire structure as an organ. ${ }^{45}$ In this concept, the enthesis is an organ that includes adjacent tendons, periosteum, and the underlying bone at attachment sites. ${ }^{8}$

\section{Bone changes}

Extensive bone disease, ranging from periostitis to osteolysis and new bone formation, is a characteristic feature of PsA, ${ }^{9}$ but radiographic studies do not permit a direct link between these findings and enthesitis. The bony point of attachment and adjacent underlying bone trabecular network forms an integral part of the enthesis organ, ${ }^{510}$ and MRI has shown that perientheseal bone oedema is an integral feature of enthesitis. ${ }^{7}$ However, it is important to point out that inflammation of the soft tissue components of large insertions is associated with bone changes in less than $50 \%$ of the cases. ${ }^{7}$ The close and interdependent relation between the insertion and adjacent bone is the basis for the diffuse perientheseal osteitis, characteristic of the SpAs and PsA, which appears to extend to diverse sites including the sacroiliac joints, the spine, and the sternoclavicular joint in the SAPHO syndrome (fig 1). It has been suggested that all fibrocartilaginous synovial joints share the same histology, biomechanics, histopathology, and imaging features as the enthesis proper and can be viewed as functional entheses (see fig 1). ${ }^{4}$ It is noteworthy that the diffuse perientheseal osteitis process is pronounced at sites devoid of synovium. ${ }^{5}$

\section{RELATION BETWEEN ENTHESITIS AND SYNOVITIS}

Compared with the other spondyloarthropathies, synovitis is especially common in PsA and has been viewed as independent from enthesitis. However, a number of MRI studies have shown a link between enthesitis and synovitis in individual swollen joints in PsA and SpA but not in RA. ${ }^{11}$ This relation has been more clearly shown in the knee joint ${ }^{10}$ but less clearly demonstrated, ${ }^{11}$ or not demonstrated, in the small joints of the hands. ${ }^{12}$ The failure of MRI to clearly distinguish this finding in some studies may be linked to a number of factors, including spatial resolution limitations of MRI for small joints, the close proximity of insertions to the synovium, and cartilage pannus regions (fig 2).

A number of ultrasound studies have also shown that clinically unrecognised enthesitis is common in the lower limbs including those insertions amenable to sonographic assessment adjacent to the knee joint in patients with PsA. ${ }^{13-16}$ Changes include thickening and oedema of the insertions, increased vascularity, bone erosion, and new bone formation. ${ }^{13-16}$

The considerable forces transmitted through the enthesis and related structures may make these sites more prone to injury than the synovium. Entheses show differences from synovial tissue that make them more comparable to articular cartilage.

Abbreviations: $M R I$, magnetic resonance imaging; $\mathrm{PsA}_{\mathrm{A}}$, psoriatic arthritis; RA, rheumatoid arthritis; SpA, spondyloarthropathy 


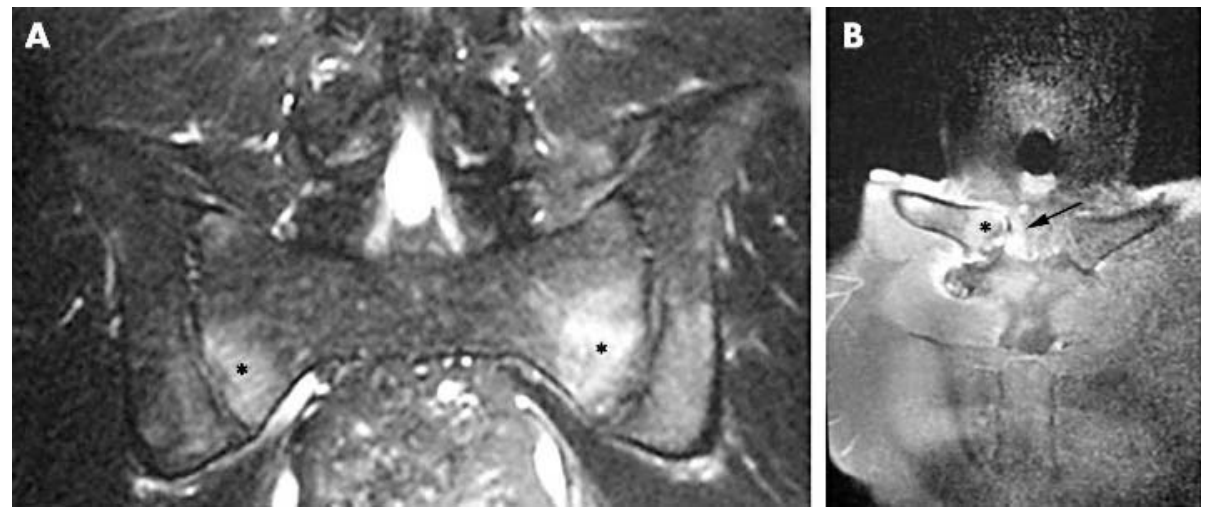

Figure 1 Magnetic resonance imaging (MRI) of the sacroiliac joint (A) and sternoclavicular joint (B) in patients with PsA. The bone adjacent to these fibrocartilaginous joints is subject to both shear and compressive forces with the MRI changes in the bone at these sites being virtually identical to that seen in bone adjacent to the fibrocartilages of large entheses such as Achilles' tendon and plantar fascia. (A) Coronal fat suppressed MRI scan of the sacroiliac joint of a male with psoriasis, peripheral arthritis, inflammatory back pain and negative human leucocyte antigen (HLA)-B27. Osteitis is denoted by asterisks. (B) Fat suppressed coronal MRI scan of the sternoclavicular joint in a male patient with psoriatic arthritis and the SAPHO syndrome. Bone oedema is adjacent to the joint fibrocartilage (asterisk) and joint swelling (arrow). These are examples of sites with high shear and compressive stress. It is suggested that these synovial joints behave like functional entheses in having a similar MRI pattern of disease as a typical insertion and hence the extensive bone disease that is independent of synovitis. (Images courtesy of Dr L Sutton, Calderdale Royal Hospital, Halifax, UK.)

- The enthesis, adjacent fibrocartilage, tendons, and ligaments are completely avascular (insertion proper) or comparatively avascular (tendon/ligament adjacent to enthesis) ${ }^{4}$

- The enthesis does not have a resident population of macrophages; indeed such cells are not detected in totally normal insertions. ${ }^{17}$

These anatomical and cellular differences between the enthesis and the synovium makes the recognition of

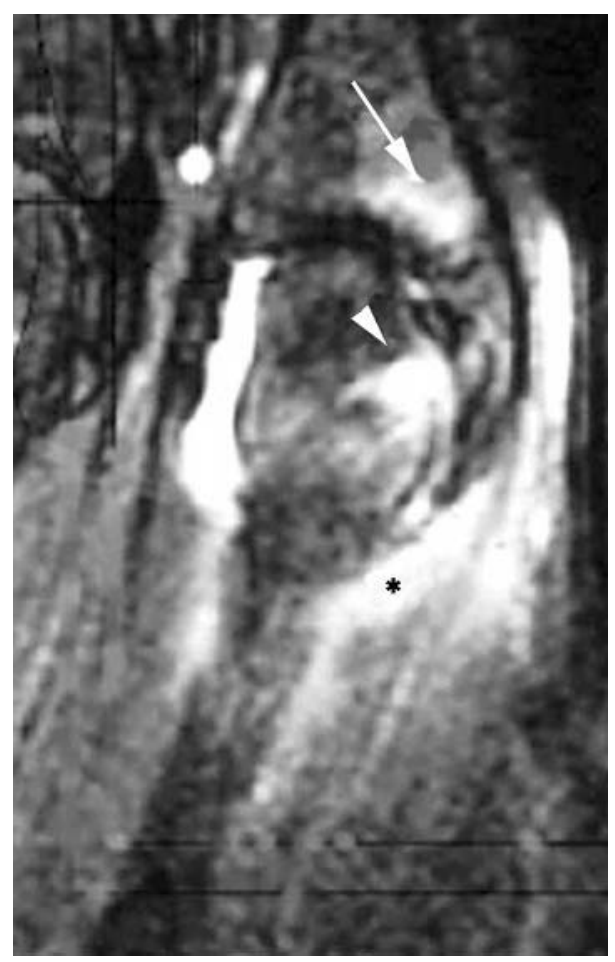

Figure 2 Fat suppressed MRI of the metacarpophalangeal (MCP) joint in a patient with psoriatic arthritis shows extracapsular inflammation (asterisk), bone oedema at the distal capsular enthesis (arrow), and bone oedema in the MCP head region where bone oedema and erosions may also occur in rheumatoid arthritis (arrowhead). enthesitis difficult, especially at sites of synovitis. Due to the intimate anatomical association of synovium and the enthesis at many sites, it would be improbable that enthesis based disease would not incite an inflammatory response within the closely juxtaposed synovial tissue, ${ }^{18}$ which is supported by the observation that resident cells in the latter can be triggered by diverse proinflammatory cues. ${ }^{18}$ Figure 3 demonstrates the close link between enthesitis and synovitis in a patient with PsA.

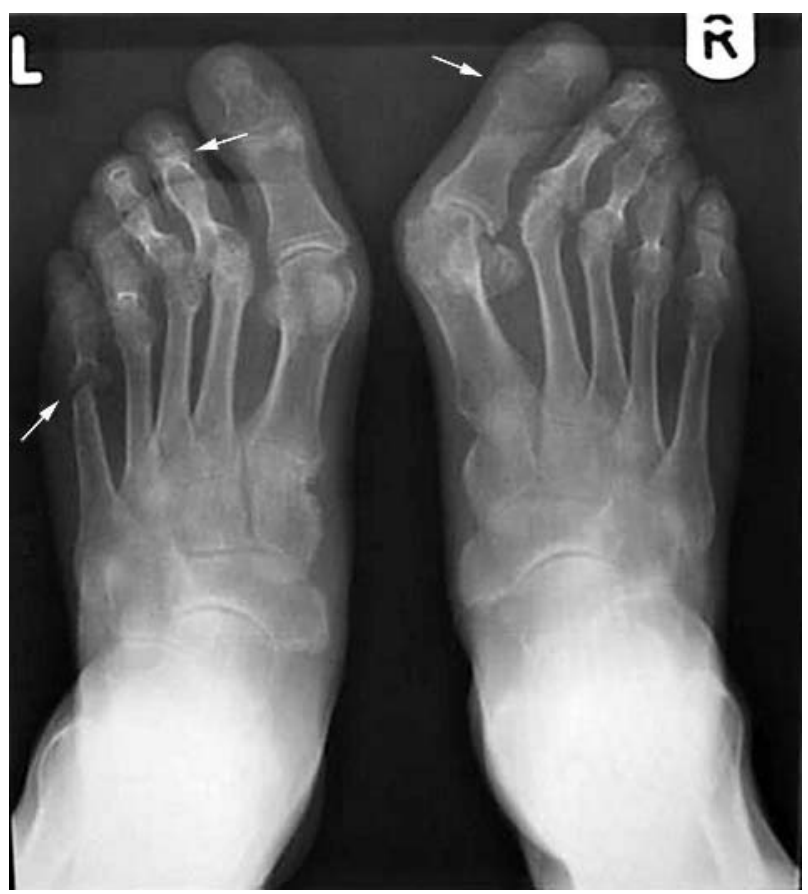

Figure 3 Radiograph of the feet of a patient with symmetrical polyarthritis with ulnar deviation of the hands and fibular deviation of the feet. The radiograph reveals joint fusion at multiple sites (arrows on top) and the fifth left metatarsal phalangeal head and adjacent proximal phalanx demonstrate pencil in cup deformity (arrow on left), features which help distinguish this as psoriatic arthritis rather than rheumatoid arthritis (RA). This case illustrates how imaging has the potential to clarify that the diagnosis is what clinically masquerades as RA. 
A hypothesis in PsA is that enthesitis arises at sites of high shear and compression forces, with the additive interaction between mechanical stress, microtrauma, tissue repair mechanisms, and bacterial molecules variably leading to inflammation. ${ }^{19}$ PsA could represent a disease where the local microenvironment response to tissue stresses partly dictates the clinical expression of disease. This model also suggests a possible explanation for the observation that PsA, on occasion, may be associated with a preceding joint injury. ${ }^{20}{ }^{21}$ Further, there may be a link to the skin component as injury to the skin is known to be a factor in expression of psoriasis. A curiosity is why psoriasis does not happen with every injury and why injury to joints of the patient with PsA associates even less frequently.

\section{THE BONE IN PsA}

From a historical perspective, bone scintigraphy showed that osteitis is a prominent feature of PsA but not RA. ${ }^{22}{ }^{23}$ This is also seen in current MRI imaging in PsA. The basis for this is incompletely understood but seems to be linked to the close functional interdependence between the enthesis, the more broadly defined "functional" enthesis, and adjacent bone.

\section{CONCLUSIONS}

Unlike RA, in which joint disease has a microanatomical basis centred on the synovium, imaging studies suggest that much of PsA pathophysiology is centred on the enthesis organ. This concept provides a unifying basis for a number of features of PsA, including disease symptoms, localisation, and imaging findings.

\section{ACKNOWLEDGEMENTS}

To all my colleagues in Leeds who make this work possible and to Prof M Benjamin in Cardiff for our ongoing fruitful collaboration on enthesis.

D McGonagle is funded by the Medical Research Council, UK

Correspondence to: Prof D McGonagle, Academic Unit Musculoskeletal Diseases, University of Leeds, and Calderdale Royal Hospital, Salterhebble, Halifax HX3 OPW, UK; d.g.mcgonagle@leeds.ac.uk

\section{REFERENCES}

1 Mease PJ. Recent advances in the management of psoriatic arthritis. Curr Opin Rheumatol 2004;16:366-70.

2 McGonagle D, Conaghan P, Emery P. Psoriatic arthritis-a unified concept 20 years on. Arthritis Rheum 1999;42:1080-6.

3 Wright V. Seronegative polyarthritis: a unified concept. Arthritis Rheum 1978;21:619-33
4 Benjamin M, McGonagle D. The anatomical basis for disease localisation in seronegative spondyloarthropathy at entheses and related sites. J Anat 2001;199:503-26.

5 McGonagle D, Marzo-Ortega H, Benjamin M, Emery P. Report on the second international enthesitis Workshop. Arthritis Rheum 2003;48:896-905.

6 McGonagle D, Gibbon W, Emery P. Classification of inflammatory arthritis by enthesitis. Lancet 1998;352:1137-40.

7 McGonagle D, Marzo-Ortega H, O'Connor P, Gibbon W, Pease C, Reece RJ, Emery P. The role of biomechanical factors and HLA-B27 on magnetic resonance imaging determined bone changes in plantar fascia enthesopathy. Arthritis Rheum 2002;46:489-43

8 Benjamin M, Moriggl B, Brenner E, Emery P, McGonagle D, Redman S. The "enthesis organ" concept: why enthesopathies may not present as focal insertional disorders. Arthritis Rheum 2004;50:3306-13.

9 Gladmann DD. Psoriatic arthritis. In: Maddison PJ, Isenberg DA, Woo P, Glass DN, eds. Oxford Textbook of Rheumatology. New York: Oxford University Press, 1998:1071-84.

10 McGonagle D, Gibbon W, O'Connor P, Green M, Pease C, Emery P. Characteristic magnetic resonance imaging entheseal changes in knee synovitis in spondylarthropathy. Arthritis Rheum 1998;41:694-700.

11 Jevtic V, Watt I, Rozman B, Kos-Golja M, Demsar F, Jarh O. Distinctive radiological features of small hand joints in rheumatoid arthritis and seronegative spondyloarthritis demonstrated by contrast-enhanced (GdDTPA) magnetic resonance imaging. Skel Radiol 1995;24:351-5.

12 Savnik A, Malmskov H, Thomsen HS, Graff LB, Nielsen H, DanneskioldSamsoe B, et al. Magnetic resonance imaging of the wrist and finger joints in patients with inflammatory joint diseases. J Rheumatol 2001;28:2193-200.

13 Frediani B, Falsetti P, Storri L, Allegri A, Bisogno S, Baldi F, Quadricepital tendon enthesitis in psoriatic arthritis and rheumatoid arthritis: ultrasound examinations and clinical correlations, et al. J Rheumatol 2001;28:2566-8.

14 Balint PV, Kane D, Wilson H, Mclnnes IB, Sturrock RD. Ultrasonography of entheseal insertions in the lower limb in spondyloarthropathy. Ann Rheum Dis 2002;61:905-10

15 D'Agostino MA, Said-Nahal R, Hacquard-Bouder C, Brasseur JL, Dougados M, Breban M. Assessment of peripheral enthesitis in the spondylarthropathies by ultrasonography combined with power Doppler: a cross-sectional study. Arthritis Rheum 2003;48:523-33.

16 Lehtinen A, Taavitsainen M, Leirisalo-Repo M. Sonographic analysis of enthesopathy in the lower extremities of patients with spondylarthropathy. Clin Exp Rheumatol 1994;12:143-8.

17 McGonagle D, Marzo-Ortega H, O'Connor P, Gibbon W, Emery P. Histological assessment of the acute enthesitis lesion in spondyloarthropathy. Ann Rheum Dis 2002;61:534-7.

18 McGonagle D, Gibbon W, Emery P. Classification of inflammatory arthritis by enthesitis. Lancet 1998;352:1137-40.

19 McGonagle D, Stockwin L, Isaacs J, Emery P. An enthesitis based model for the pathogenesis of spondyloarthropathy. Additive effects of microbial adjuvant and biomechanical factors at disease sites. J Rheumatol 2001;28:2155-9.

20 Scarpa R, Del Puente A, di Girolamo C, della Valle G, Lubrano E, Oriente P. Interplay between environmental factors, articular involvement, and HLA-B27 in patients with psoriatic arthritis. Ann Rheum Dis 1992;51:78-9.

21 Punzi L, Pianon M, Bertazzolo N, Fagiolo U, Rizzi E, Rossini P, et al. Clinical, laboratory and immunogenetic aspects of post-traumatic psoriatic arthritis: a study of 25 patients. Clin Exp Rheumatol 1998;16:277-81.

22 Namey TC, Rosenthall L. Periarticular uptake of ${ }^{99 m_{\text {technetium diphosphonate }}}$ in psoriatics: correlation with cutaneous activity. Arthritis Rheum 1976:19:607-12.

23 Helliwell P, Marchesoni A, Peters M, Barker M, Wright V. A re-evaluation of the osteoarticular manifestations of psoriasis. Br J Rheumatol $1991 ; 30: 339-45$ 